\title{
Prática profissional no contexto hospitalar: visão de enfermeiros sobre contribuições das concepções de Dorothea Orem
}

\author{
Professional practice in the hospital context: nurses' view on the contributions of Dorothea Orem's \\ conceptions
}

La práctica profesional en el contexto hospitalario: la visión de las enfermeras sobre las contribuciones de las concepciones de Dorothea Orem

\section{Olga Maria Pimenta Lopes Ribeiro', Letícia de Lima Trindade ${ }^{\mathrm{II}}$, João Miguel Almeida Ventura da Silva ${ }^{\text {III }}$, Ana da Conceição Alves Faria ${ }^{\text {IV }}$}

\begin{abstract}
Resumo: Objetivo: analisar a concordância dos enfermeiros sobre as contribuições das concepções de Dorothea Orem para a prática profissional no contexto hospitalar. Método: estudo quantitativo, transversal, realizado em 36 hospitais portugueses, com participação de 3451 enfermeiros. A coleta de dados decorreu entre julho de 2015 e março de 2016, por meio de questionário. Para análise, usou-se estatística descritiva e analítica. Resultados: confirmou-se que 75 a 85\% dos participantes, consideraram que as concepções de Orem com relação aos quatro conceitos metaparadigmáticos, estão de acordo ou totalmente de acordo com a sua prática. A Região do país, o serviço e a condição em que se exerce a profissão foram as variáveis que influenciaram a concordância com as concepções. Conclusão: a conformidade observada nos resultados quanto à concordância dos enfermeiros com os conceitos metaparadigmáticos, reforça o contributo do referencial de Orem para a sustentação da prática e para a garantia da cientificidade da profissão.
\end{abstract}

Descritores: Enfermagem; Teoria de enfermagem; Modelos de enfermagem; Prática Profissional; Hospitais

\begin{abstract}
Objective: to analyze the concordance of nurses about the contributions of Dorothea Orem's conceptions to professional practice in the hospital context. Method: a quantitative cross-sectional study conducted in 36 Portuguese hospitals, with the participation of 3451 nurses. Data was collected using a questionnaire between July 2015 and March 2016. For analysis, descriptive and analytical statistics were used. Results: it was confirmed that 75 to $85 \%$ of the participants considered that Orem's conceptions regarding the four
\end{abstract}

\footnotetext{
I Professora Adjunta, Doutora em Ciências de Enfermagem, Escola Superior de Enfermagem do Porto. Porto, Portugal. E-mail: olgaribeiro@esenf.pt, Orcid: https://orcid.org/0000-0001-9982-9537.

II Professora Adjunta, Doutora em Enfermagem, Universidade do Estado de Santa Catarina e Universidade Comunitária da Região de Chapecó. Chapecó, Santa Catarina, Brasil. E-mail: letrindade@hotmail.com, Orcid: https://orcid.org/0000-0002-7119-0230

III Enfermeiro, Mestre em Ciências de Enfermagem, Centro Hospitalar Universitário de São João. Porto, Portugal. E-mail: joao.miguel.silva@chsj.min-saude.pt, Orcid: https://orcid.org/0000-0002-8794-528X

IV Enfermeira, Mestre em Enfermagem de Reabilitação, Administração Regional de Saúde do Norte, Agrupamento de Centros de Saúde Ave/Famalicão. Vila Nova de Famalicão, Portugal. E-mail: acafaria@arsnorte.min-saude.pt, Orcid: https://orcid.org/0000-0002-5838-0080
} 
Prática profissional no contexto hospitalar: visão de enfermeiros sobre contribuições... $\mid 2$

meta-paradigmatic concepts are in concordance or totally in concordance with their practice. The Region of the country, the service, and the condition in which the profession is practiced were the variables that influenced the concordance with the conceptions. Conclusion: the conformity observed in the results regarding the concordance of the nurses with the meta-paradigmatic concepts reinforces the contribution of Orem's framework to support the practice and to guarantee the scientificity of the profession.

Descriptors: Nursing; Nursing Theory; Models, Nursing; Professional Practice; Hospitals

Resumen: Objetivo: analizar el acuerdo de enfermeros sobre las contribuciones de las concepciones de Dorothea Orem para la práctica profesional en el contexto hospitalario. Método: estudio cuantitativo, transversal, realizado en 36 hospitales portugueses, con la participación de 3451 enfermeros. La recolección de datos se llevó a cabo entre julio de 2015 y marzo de 2016, mediante un cuestionario. Para el análisis se utilizó estadística descriptiva y analítica. Resultados: se constató que 75 a $85 \%$ de los participantes consideraron que las concepciones de Orem sobre los cuatro conceptos metaparadigmáticos, están de acuerdo o totalmente de acuerdo con su práctica. La región del país, el servicio y la condición en la que se ejerce la profesión fueron las variables que influyeron en el acuerdo con las concepciones. Conclusión: la conformidad observada en los resultados sobre la concordancia de los enfermeros con los conceptos metaparadigmáticos, refuerza el aporte del marco de Orem para sostener la práctica y garantizar la cientificidad de la profesión.

Descriptores: Enfermería; Teoría de Enfermería; Modelos de Enfermería; Práctica Profesional; Hospitales

\section{Introdução}

$\mathrm{Na}$ Enfermagem, ultrapassado o tempo em que os princípios científicos, eram fundamentalmente provenientes de outras ciências, tem sido notório o empenho dos enfermeiros em reconhecer e tornar público o seu saber próprio. ${ }^{1-2}$ Além disso, ao longo dos últimos anos, tornou-se consensual, entre vários autores, que sustentar a prática de enfermagem apenas no modelo biomédico, sem incorporar conhecimento próprio, essencialmente por meio das teorias de enfermagem, não amplia nem sedimenta a atuação específica dos enfermeiros..$^{3-4}$

Diante da complexidade do exercício profissional, as teorias de enfermagem são capazes de produzir descrições e explicações consistentes dos fenómenos, sendo absolutamente determinantes nas predições e prescrições dos enfermeiros. ${ }^{1,3}$ Embora recentemente alguns autores advirtam para o potencial das teorias, o valor que lhes tem sido atribuído, e a sua incorporação na prática clínica são, muitas vezes, inferiores ao expectável. ${ }^{1-2}$

$\mathrm{Na}$ certeza de que teoria e prática devem caminhar lado a lado, as teorias de enfermagem podem ser determinantes na criação de pontes, entre os enfermeiros “práticos” e acadêmicos, 
contribuindo para a anulação da ideia de que o conhecimento é apenas produzido pelos enfermeiros doutores e consumido pelos enfermeiros "práticos”. ${ }^{3}$ Embora se reconheça que nem todas as teorias se adequam às diversas realidades contextuais, cabe aos enfermeiros identificar as que melhor se ajustam às demandas do cuidado profissional, considerando o seu potencial para sustentar o exercício profissional..$^{2-3}$

Acresce que o cuidado de enfermagem para ser implementado com qualidade deve estar ancorado nas teorias de enfermagem, ${ }^{5-6}$ que com diferentes olhares permitem explicar os fenômenos inerentes à prática dos enfermeiros. ${ }^{1}$ Ao fundamentar a sua atuação em teóricas da profissão, os enfermeiros conferem sentido à sua prática assistencial, com potencial diferenciação na construção do raciocínio e do julgamento clínico, bem como revelar-se mais subsidiados para definir as intervenções de enfermagem e alcançar melhores resultados. ${ }^{1}$

Atendendo à essência da Enfermagem, o referencial teórico de Dorothea Orem, com enfoque no autocuidado, tem sido um dos mais frequentemente incorporados na prática clínica. Na perspetiva da teórica, o autocuidado refere-se ao desempenho ou à prática de atividades que as pessoas realizam para manter a vida, a saúde e o bem-estar..$^{7-8}$

Além disso, o seu referencial integra três teorias: Teoria do Autocuidado (que apresenta o porquê e o modo como as pessoas cuidam de si próprias), Teoria do Déficit no Autocuidado (que apresenta e explica o motivo pelo qual as pessoas podem ser ajudadas pelos profissionais de enfermagem) e Teoria dos Sistemas de Enfermagem (que apresenta e explica como é que os enfermeiros e/ou pessoas dão resposta às necessidades de autocuidado). ${ }^{7,9-10} \mathrm{~A}$ Teoria dos Sistemas de Enfermagem é a mais externa e envolvente e contém a Teoria do Déficit no Autocuidado. Por sua vez, a Teoria do Autocuidado é um componente da Teoria do Déficit no Autocuidado. ${ }^{7}$

Tal como aconteceu em relação ao referencial teórico de Orem, à medida que foram sendo desenvolvidas, as diversas teorias de enfermagem alicerçaram a construção de uma base de conhecimento sólida, organizando o mundo fenomenal da Enfermagem em torno daqueles 
Prática profissional no contexto hospitalar: visão de enfermeiros sobre contribuições... $\mid 4$

que ficaram amplamente reconhecidos como conceitos metaparadigmáticos: enfermagem, pessoa, saúde e ambiente..$^{3,11}$

Nesta perspetiva, importa considerar que para selecionar a teoria que melhor corresponderá às exigências de um cuidado individualizado e intencional, é essencial que haja congruência entre os conceitos metaparadigmáticos e a atuação dos enfermeiros nos diferentes contextos clínicos. ${ }^{12}$ Assim, quando se reflete na relevância de uma determinada teoria para a sustentação da prática profissional, torna-se importante indagar a concordância dos enfermeiros sobre os seus conceitos centrais, com especial destaque para a enfermagem, a pessoa, a saúde e o ambiente.

Partindo destes pressupostos, no âmbito de uma investigação mais ampla, realizada em Portugal, no contexto nacional, e intitulada "Contextos da prática hospitalar e concepções dos enfermeiros”, ${ }^{13}$ além de outros, estabeleceu-se como objetivo, identificar o grau de concordância dos enfermeiros sobre os conceitos metaparadigmáticos, à luz de 13 teóricas de enfermagem, e a sua aplicação na prática profissional. A falta de estudos sobre a temática e, consequentemente, o desconhecimento quanto aos referenciais teóricos com que os enfermeiros portugueses mais se identificavam, constituíram os principais desafios para a realização dessa pesquisa. Empiricamente era conhecido que em Portugal, o refencial teórico de Dorothea Orem é um dos que mais se adequa ao exercício profissional dos enfermreiros, no entanto, nenhuma investigação prévia tinha deixado evidente tal fato.

Assim, esta pesquisa pretende responder ao seguinte questionamento: Qual a concordância dos enfermeiros, sobre as contribuições das concepções de enfermagem, pessoa, saúde e ambiente de Dorothea Orem, para a prática profissinal no contexto hospitalar?

Diante do referido, neste artigo objetivou-se analisar a concordância dos enfermeiros sobre as contribuições das concepções de Dorothea Orem para a prática profissional no contexto hospitalar. 
5 | Ribeiro OMPL, Trindade LL, Silva JMAV, Faria ACA

\section{Método}

Estudo quantitativo, transversal, realizado em 36 instituições hospitalares de Portugal continental. A técnica de amostragem usada foi não probabilística por conveniência. A partir de uma população acessível de 10.013 enfermeiros, para um intervalo de confiança de 95\% e um nível de significância de 5\%, obteve-se uma amostra de 3,451 enfermeiros. Como critérios de inclusão definiram-se: exercer a atividade profissional no contexto hospitalar pelo menos há seis meses, nos serviços dos departamentos de medicina, cirurgia e medicina intensiva e urgência. Foram excluídos os enfermeiros que no período da coleta de dados estavam de licença, independemente do motivo.

Como instrumento de coleta de dados foi utilizado um questionário de autopreenchimento, organizado em duas partes: a primeira com a caracterização sociodemográfica e profissional dos participantes e, a segunda, relativa às concepções de enfermagem, pessoa, saúde e ambiente. Embora no âmbito da investigação realizada em Portugal, no contexto nacional, ${ }^{13}$ no instrumento estivessem claramente expressas as concepções de 13 teóricas de enfermagem em relação aos conceitos metaparadigmáticos, atendendo ao objetivo deste estudo será dado enfoque às concepções de Dorothea Orem.

Aquando do preenchimento do questionário era pedido aos participantes que respondessem de acordo com a sua concordância com as concepções de Orem sobre enfermagem, pessoa, saúde e ambiente, sendo que numa escala do tipo Likert as respostas poderiam variar entre 1 “está totalmente em desacordo com a minha prática”, 2 “está em desacordo com a minha prática”, 3 "não tenho opinião", 4 "está de acordo com a minha prática” e 5 “está totalmente de acordo com a minha prática”.

Importa referir que o questionário usado foi construído pelos próprios investigadores, e validado pelos mesmos em estudo piloto, prévio a esta investigação, com participação de enfermeiros de outros hospitais. Na primeira fase da concepção desse instrumento, a validação 
Prática profissional no contexto hospitalar: visão de enfermeiros sobre contribuições... $\mid 6$

de conteúdo foi efetuada por peritos, estudiosos dos referenciais teóricos de enfermagem e, posteriormente, para validação de constructo e consistência interna, empregou-se a análise fatorial exploratória e o coeficiente alfa de Cronbach.

Depois de confirmada a validade e confiabilidade do instrumento, a coleta de dados, efetuada por um dos investigadores, ocorreu entre julho de 2015 e março de 2016, após aprovação das Comissões de Ética e dos Conselhos de Administração dos 36 hospitais onde se realizou o estudo. Em cada serviço das áreas incluídas, foram entregues os questionários correspondentes ao número de enfermeiros que atuavam nos setores e, posteriormente, recolhidos no local, mediante agendamento prévio e disponibilidade dos profissionais. Além da informação escrita, que estava anexa ao questionário, nesse momento, também foi apresentada presencialmente a pesquisa aos enfermeiros. Após o esclarecimento sobre os objetivos, os enfermeiros tinham a liberdade de preencher ou não o questionário, colocando-o posteriormente num envelope fechado.

No tratamento dos dados foi usado o Statistical Package for the Social Sciences (SPSS), versão 22.0. Aquando da análise dos resultados, considerou-se o nível de significância de 0,05 e um nível de confiança de 0,95 .

Para compreender a distribuição das variáveis, considerando a sua natureza, foram utilizados o teste Wilcoxon-Mann-Whitney e o teste de Kruskal-Wallis. Para confirmar a existência de diferenças na localização das distribuições, recorreu-se à distribuição qui-quadrado. Atendendo a que o grau de concordância dos participantes com as concepções, é uma variável qualitativa ordinal, para identificar as variáveis de caracterização que afetavam o grau de concordância, foram usados modelos de regressão logística acumulados para respostas ordinais.

Com recurso a esses modelos, numa primeira fase, foram identificadas as variáveis explicativas que influenciavam o grau de concordância com as concepções de enfermagem, pessoa, saúde e ambiente. Posteriormente, para selecionar as variáveis explicativas a reter no 
modelo, efetuou-se uma seleção “backward" baseada no teste de Wald, eliminando-se as variáveis cujo parâmetro estimado não era estatisticamente significativo. As variáveis de caraterização contempladas nesta análise foram a Região do país, o serviço, o gênero, o grau acadêmico, a condição em que se exerce a profissão e o tempo de exercício profissional.

Importa referir que esta pesquisa, integrada em uma investigação nacional, foi conduzida de acordo com os padrões éticos exigidos, tendo sido previamente aprovada por uma Comissão de Ética de um Hospital da Região Norte de Portugal, no dia 13 de maio de 2015, com o número 98-15. Todos os enfermeiros que participaram no estudo, assinaram o consentimento informado, tendo sido garantida a confidencialidade e o anonimato das informações coletadas.

\section{Resultados}

Com relação às características sociodemográficas e profissionais, os 3451 participantes, eram maioritariamente do sexo feminino ( $\mathrm{n}=2661)$, com uma idade média de 36,4 anos e desvio padrão de 8,3. No que se refere ao estado civil, 2109 eram casados ou viviam em união de facto, 1168 solteiros, 163 divorciados e 11 viúvos.

No que concerne à condição em que exercem a profissão, 2633 eram enfermeiros de cuidados gerais, 686 enfermeiros especialistas e 132 enfermeiros gestores. Relativamente às áreas de especialização, 306 eram especialistas em enfermagem de reabilitação, 259 em enfermagem médico-cirúrgica, 55 em enfermagem comunitária, 37 em enfermagem de saúde mental e psiquiatria, 17 em enfermagem de saúde infantil e pediatria, e, por fim, 12 em enfermagem de saúde materna e obstetrícia.

Com relação ao grau acadêmico, 3037 eram licenciados, 369 mestres, 38 possuíam o bacharelado e 7 o doutorado. No que se refere à distribuição dos enfermeiros segundo as Administrações Regionais de Saúde (ARS) que integram os hospitais onde se realizou o estudo, 
Prática profissional no contexto hospitalar: visão de enfermeiros sobre contribuições... $\mid 8$

prevaleceu o Norte com 1492 participantes, Lisboa e Vale do Tejo com 829, o Centro com 771, o Alentejo com 213 e o Algarve com 146.

Atendendo a que as instituições hospitalares estão distribuídas em Portugal continental por cinco Regiões, que correspondem às já mencionadas Administrações Regionais de Saúde, os resultados relativos às concepções de enfermagem, pessoa, saúde e ambiente serão apresentados conforme esses territórios.

\section{Concordância com as concepções de enfermagem, pessoa, saúde e ambiente de Dorothea Orem}

No que se refere à conceção de enfermagem de Dorothea Orem, embora a opção “de acordo com a minha prática” constituísse a resposta mais frequente, $32,6 \%$ dos participantes da Região Centro e $29,8 \%$ da Região Norte afirmaram que essa conceção está "totalmente de acordo com a sua prática” (Tabela 1).

Tabela 1 - Concordância dos enfermeiros com a concepção de enfermagem de Dorothea Orem, nas cinco Regiões do país. Porto, Portugal, 2017

\begin{tabular}{rcccccc}
\hline & \multicolumn{6}{c}{ Concordância com a concepção de enfermagem de Dorothea Orem } \\
\cline { 2 - 7 } Região do País & $1^{*}$ & $2^{+}$ & $3^{+}$ & $4^{\S}$ & $5^{\|}$ & Total \\
& $\mathbf{n}(\%)$ & $\mathbf{n}(\%)$ & $\mathbf{n}(\%)$ & $\mathbf{n}(\%)$ & $\mathbf{n}(\%)$ & $\mathbf{n}(\%)$ \\
\hline Norte & $20(1,3)$ & $102(6,8)$ & $169(11,3)$ & $757(50,7)$ & $444(29,8)$ & $1492(100)$ \\
Centro & $3(0,4)$ & $14(1,8)$ & $119(15,4)$ & $384(49,8)$ & $251(32,6)$ & $771(100)$ \\
Lisboa e Vale do Tejo & $7(0,8)$ & $52(6,3)$ & $138(16,6)$ & $467(56,3)$ & $165(19,9)$ & $829(100)$ \\
Alentejo & $1(0,5)$ & $7(3,3)$ & $38(17,8)$ & $116(54,5)$ & $51(23,9)$ & $213(100)$ \\
Algarve & $0(0,0)$ & $0(0,0)$ & $21(14,4)$ & $98(67,1)$ & $27(18,5)$ & $146(100)$ \\
\hline
\end{tabular}

Legenda: $1^{*}$ - Totalmente em desacordo com a minha prática; $2^{+}$- Em desacordo com a minha prática; $3^{*}$ - Sem opinião; $4^{\S}$ - De acordo com a minha prática; $5^{\|}$- Totalmente de acordo com a minha prática Fonte: Elaboração própria, dados da pesquisa, $2017 .{ }^{13}$

Após a análise descritiva, com recurso ao teste de Kruskal-Wallis, confirmaram-se associações significativas entre as Regiões e a concepção de enfermagem, com Estatística do teste de 37,2 e p < 0,001. Consequentemente, tornou-se necessário efetuar comparações múltiplas, entre todos os pares de Regiões. Neste sentido, usando o teste Wilcoxon-Mann-Whitney para amostras 
independentes, com recurso à correção de Bonferroni, e com um nível de significância corrigido de $0,5 \%$, identificou-se que a concepção de enfermagem de Orem está mais de acordo com a prática dos enfermeiros das Regiões Norte e Centro do que em Lisboa e Vale do Tejo, estando igualmente de acordo com a prática dos participantes em todos os outros pares de Regiões.

No que se refere à concepção de pessoa de Dorothea Orem, embora a opção "de acordo com a minha prática” fosse a resposta majoritária, $32,4 \%$ dos participantes do Centro e $27,9 \%$ do Norte afirmaram que essa conceção está “totalmente de acordo com a sua prática” (Tabela 2).

Tabela 2 - Concordância dos enfermeiros com a concepção de pessoa de Dorothea Orem, nas cinco Regiões do país. Porto, Portugal, 2017

\begin{tabular}{rcccccc}
\hline & \multicolumn{6}{c}{ Concordância com a concepção de pessoa de Dorothea Orem } \\
\cline { 2 - 7 } Região do País & $1^{*}$ & $2^{+}$ & $3^{*}$ & $4^{\S}$ & $5^{\|}$ & Total \\
& $\mathbf{n}(\%)$ & $\mathbf{n}(\%)$ & $\mathbf{n}(\%)$ & $\mathbf{n}(\%)$ & $\mathbf{n}(\%)$ & $\mathbf{n}(\%)$ \\
\hline Norte & $5(0,3)$ & $42(2,8)$ & $223(14,9)$ & $805(54,0)$ & $417(27,9)$ & $1492(100)$ \\
Centro & $1(0,1)$ & $8(1,0)$ & $114(14,8)$ & $398(51,6)$ & $250(32,4)$ & $771(100)$ \\
Lisboa e Vale do Tejo & $0(0,0)$ & $28(3,4)$ & $138(16,6)$ & $489(59,0)$ & $174(21,0)$ & $829(100)$ \\
Alentejo & $0(0,0)$ & $5(2,3)$ & $40(18,8)$ & $113(53,1)$ & $55(25,8)$ & $213(100)$ \\
Algarve & $0(0,0)$ & $1(0,7)$ & $14(9,6)$ & $102(69,9)$ & $29(19,9)$ & $146(100)$ \\
\hline
\end{tabular}

Legenda: $1^{*}$ - Totalmente em desacordo com a minha prática; $2^{+}$- Em desacordo com a minha prática; $3^{*}$ - Sem opinião; $4^{\S}$ - De acordo com a minha prática; $5^{\|}$- Totalmente de acordo com a minha prática Fonte: Elaboração própria, dados da pesquisa, $2017 \cdot{ }^{13}$

Depois da análise descritiva, utilizando o teste de Kruskal-Wallis, verificaram-se associações significativas entre as Regiões e a conceção de pessoa, com Estatística do teste de 24,8 e p $<0,001$. Uma vez que existiam diferenças entre as Regiões, procedeu-se a comparações múltiplas, entre todos os pares de Regiões, usando o teste Wilcoxon-MannWhitney para amostras independentes, com recurso à correção de Bonferroni, e com um nível de significância corrigido de $0,5 \%$, tendo-se concluído que a conceção de pessoa de Orem está também mais de acordo com a prática dos enfermeiros das Regiões Norte e 
Prática profissional no contexto hospitalar: visão de enfermeiros sobre contribuições... $\mid 10$

Centro do que em Lisboa e Vale do Tejo, estando igualmente de acordo com a prática dos participantes em todos os outros pares de Regiões.

No que concerne à concepção de saúde de Dorothea Orem, embora a opção "de acordo com a minha prática” fosse a resposta predominante, $29,2 \%$ dos participantes da Região Centro e $28,3 \%$ da Região Norte afirmaram que essa concepção está “totalmente de acordo com a sua prática” (Tabela 3).

Tabela 3 - Concordância dos enfermeiros com a concepção de saúde de Dorothea Orem, nas cinco Regiões do país. Porto, Portugal, 2017

\begin{tabular}{rcccccc}
\hline & \multicolumn{5}{c}{ Concordância com a concepção de saúde de Dorothea Orem } \\
\cline { 2 - 7 } Região do País & $1^{*}$ & $2^{+}$ & $3^{*}$ & $4^{\S}$ & $5^{\|}$ & Total \\
& $\mathbf{n}(\%)$ & $\mathbf{n}(\%)$ & $\mathbf{n}(\%)$ & $\mathbf{n}(\%)$ & $\mathbf{n}(\%)$ & $\mathbf{n}(\%)$ \\
\hline Norte & $8(0,5)$ & $65(4,4)$ & $206(13,8)$ & $791(53,0)$ & $422(28,3)$ & $1492(100)$ \\
Centro & $1(0,1)$ & $9(1,2)$ & $124(16,1)$ & $412(53,4)$ & $255(29,2)$ & $771(100)$ \\
Lisboa e Vale do Tejo & $5(0,6)$ & $30(3,6)$ & $139(16,8)$ & $515(62,1)$ & $140(16,9)$ & $829(100)$ \\
Alentejo & $0(0,0)$ & $9(4,2)$ & $45(21,1)$ & $105(49,3)$ & $54(25,4)$ & $213(100)$ \\
Algarve & $0(0,0)$ & $0(0,0)$ & $24(16,4)$ & $95(65,1)$ & $27(18,5)$ & $146(100)$ \\
\hline
\end{tabular}

Legenda: $1^{*}$ - Totalmente em desacordo com a minha prática; $2^{*}$ - Em desacordo com a minha prática; $3^{*}$ - Sem opinião; $4^{\S}$ - De acordo com a minha prática; $5^{\|}$- Totalmente de acordo com a minha prática Fonte: Elaboração própria, dados da pesquisa, 2017.13

Após a análise descritiva, com recurso ao teste de Kruskal-Wallis, confirmaram-se associações significativas entre as Regiões e a concepção de saúde, com Estatística do teste de 32,9 e p $<0,001$. Dado que existiam diferenças entre as Regiões, usando o teste Wilcoxon-MannWhitney para amostras independentes, com recurso à correção de Bonferroni, e com um nível de significância corrigido de $0,5 \%$, procedeu-se a comparações múltiplas, entre todos os pares de Regiões, tendo-se concluído que a concepção de saúde está também mais de acordo com a prática dos enfermeiros das Regiões Norte e Centro do que em Lisboa e Vale do Tejo, estando igualmente de acordo com a prática dos participantes em todos os outros pares de Regiões.

No que concerne à concepção de ambiente de Dorothea Orem, embora a opção “de acordo com a minha prática” continuasse a ser a resposta mais frequente, $33,2 \%$ dos 
participantes do Centro e 32,9\% do Alentejo afirmaram que essa conceção está "totalmente de acordo com a sua prática” (Tabela 4).

Tabela 4 - Concordância dos enfermeiros com a concepção de ambiente de Dorothea Orem, nas cinco Regiões do país. Porto, Portugal, 2017

\begin{tabular}{rcccccc}
\hline \multirow{2}{*}{ Região do País } & \multicolumn{6}{c}{ Concordância com a concepção de ambiente de Dorothea Orem } \\
\cline { 2 - 7 } & $1^{*}$ & $2^{*}$ & $3^{*}$ & $4^{\S}$ & $5^{\|}$ & Total \\
& $\mathbf{n}(\%)$ & $\mathbf{n}(\%)$ & $\mathbf{n}(\%)$ & $\mathbf{n}(\%)$ & $\mathbf{n}(\%)$ & $\mathbf{n}(\%)$ \\
\hline Norte & $6(0,4)$ & $27(1,8)$ & $136(9,1)$ & $890(59,7)$ & $443(29,0)$ & $1492(100)$ \\
Centro & $0(0,0)$ & $7(0,9)$ & $73(9,5)$ & $435(56,4)$ & $256(33,2)$ & $771(100)$ \\
Lisboa e Vale do Tejo & $3(0,4)$ & $9(1,1)$ & $63(7,6)$ & $525(63,3)$ & $229(27,6)$ & $829(100)$ \\
Alentejo & $1(0,5)$ & $5(2,3)$ & $26(12,2)$ & $111(52,1)$ & $70(32,9)$ & $213(100)$ \\
Algarve & $0(0,0)$ & $0(0,0)$ & $12(8,2)$ & $99(67,8)$ & $35(24,0)$ & $146(100)$ \\
\hline
\end{tabular}

Legenda: $1^{*}$ - Totalmente em desacordo com a minha prática; $2^{+}$- Em desacordo com a minha prática; $3^{*}$ - Sem opinião; $4^{\S}$ - De acordo com a minha prática; $5^{\|}$- Totalmente de acordo com a minha prática Fonte: Elaboração própria, dados da pesquisa, $2017 .{ }^{13}$

Depois da análise descritiva, confirmou-se com recurso ao teste de Kruskal-Wallis, que não existiam associações significativas entre as regiões e a conceção de ambiente de Dorothea Orem, com Estatística do teste de 5,1 e p $<0,281$. Neste sentido, o grau de concordância com a conceção de ambiente é o mesmo em todas as Regiões.

\section{Características sociodemográficas e profissionais e concepções de Dorothea Orem}

Usando os modelos de regressão logística de multicategoria, verificou-se que nas concepções de enfermagem, pessoa e saúde, as variáveis explicativas retidas nos respetivos modelos, isto é, as que afetaram o grau de concordância com estas concepções foram a Região do país, o serviço e a condição em que se exerce a profissão.

No caso da concepção de enfermagem, o grau de concordância foi maior nos enfermeiros que trabalham no Centro e menor nos que trabalham em Lisboa e Vale do Tejo. Numa posição intermédia, ficou a concordância dos participantes das Regiões Norte, Alentejo ou Algarve. 
Prática profissional no contexto hospitalar: visão de enfermeiros sobre contribuições... $\mid 12$

Relativamente ao serviço, a Medicina Intensiva e Urgência foi o contexto clínico onde se registou menor grau de concordância. Quanto à condição em que se exerce a profissão, o grau de concordância foi superior nos enfermeiros especialistas.

$\mathrm{Na}$ concepção de pessoa, o grau de concordância também foi maior nos profissionais de enfermagem que trabalham no Centro e menor nos que trabalham em Lisboa e Vale do Tejo. Em uma posição intermédia, voltou a ficar a concordância dos que exercem funções nas Regiões Norte, Alentejo ou Algarve. A Medicina e Especialidades Médicas foi o contexto clínico que registou maior concordância, seguindo-se a Cirurgia e Especialidades Cirúrgicas e, posteriormente, a Medicina Intensiva e Urgência, em que a concordância foi menor. Por fim, foram os enfermeiros especialistas os que apresentaram maior grau de concordância.

$\mathrm{Na}$ concepção de saúde, os participantes que trabalham em Lisboa e Vale do Tejo, e em contextos de Medicina Intensiva e Urgência, apresentaram menor grau de concordância do que os enfermeiros de qualquer outra Região ou serviço. Além disso, os enfermeiros especialistas foram os que apresentaram maior grau de concordância.

Em relação à concepção de ambiente, evidenciou-se que além da Região, do serviço e da condição em que se exerce a profissão, o gênero também afetou o grau de concordância. Quanto à Região do país, os participantes que trabalham no Centro apresentaram maior grau de concordância do que os enfermeiros que trabalham em qualquer outra Região. Os enfermeiros que trabalham na Medicina Intensiva e Urgência apresentaram grau de concordância mais baixo. Os enfermeiros especialistas continuaram a revelar maior grau de concordância. Por último, o grau de concordância dos enfermeiros foi inferior ao das enfermeiras.

A Figura 1 resume as variáveis de caracterização que traduzem maior concordância com as concepções de Dorothea Orem. 


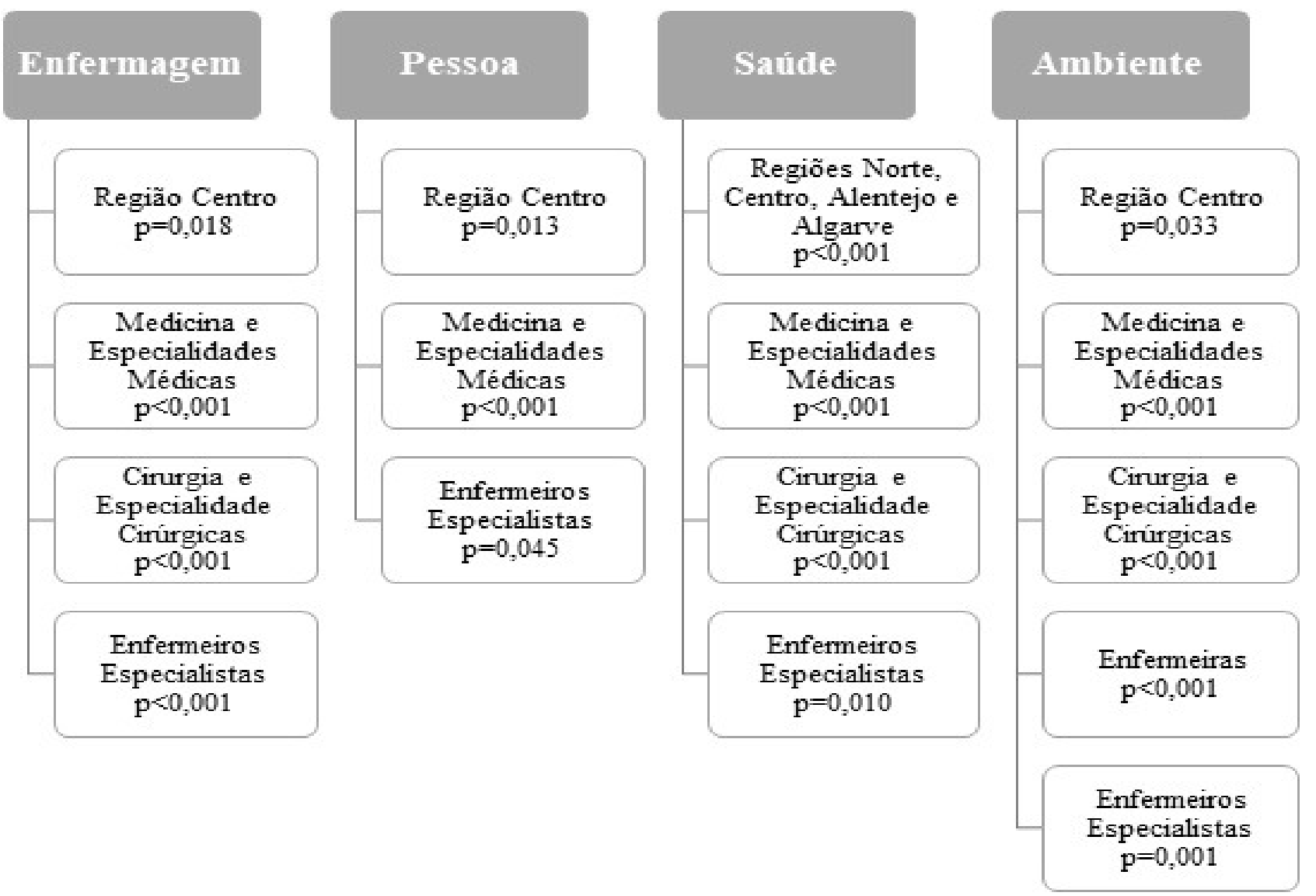

Figura 1 - Variáveis de caracterização que traduzem maior concordância dos enfermeiros com as concepções de Dorothea Orem para os conceitos metaparadigmáticos

Fonte: Elaboração própria, dados da pesquisa, $2017 .{ }^{13}$

\section{Discussão}

O alinhamento das teorias à prática profissional de enfermagem torna-se cada vez mais fundamental ao alcance de boas práticas e, consequentemente, à melhoria da qualidade da assistência prestada. ${ }^{2-3,14-15}$ Para serem facilmente integradas na prática, as teorias de enfermagem devem obedecer a três critérios: serem aplicáveis na prática profissional, serem úteis e compatíveis com o contexto multiprofissional e multidisciplinar, característico da área da saúde e ter relevância para o cuidado a prestar ao cliente. ${ }^{3}$ Com enfoque num dos conceitos centrais da enfermagem - o autocuidado - o referencial teórico de Dorothea Orem corresponde favoravelmente a todos esses critérios. 
Prática profissional no contexto hospitalar: visão de enfermeiros sobre contribuições... | 14

$\mathrm{Na}$ perspetiva de diversos autores, a pertinência deste referencial está no seu potencial para sustentar a prática clínica, dando particular enfoque ao cliente, na medida em que o considera capaz de aprender novas formas de concretizar o autocuidado. , $, 10,14^{2}$

Em todas as regiões de Portugal, quando analisada a concordância dos enfermeiros em relação aos conceitos metaparadigmáticos, à luz de 13 teóricas de enfermagem, constatou-se que as concepções de Virginia Henderson, Afaf Meleis e Dorothea Orem foram as que obtiveram maior concordância. ${ }^{12}$

$\mathrm{Na}$ sequência do valor atribuído pelos enfermeiros ao autocuidado e mantendo, no âmbito do exercício profissional dos enfermeiros portugueses, o destaque na "Escola das Necessidades" ${ }^{12}$ é de salientar que neste estudo uma percentagem significativa de participantes (75 a 85\%) de todas as Regiões do país, qualificaram as concepções de Dorothea Orem como de acordo ou totalmente de acordo com a sua prática.

O mencionado vem revelar que nos contextos da prática, a perspetiva de Orem tem orientado a ação dos enfermeiros, que de forma deliberada e sistemática assistem os clientes com dependência, na concretização das atividades inerentes ao autocuidado. ${ }^{2,15-16} \mathrm{~A}$ influência da formação e das instituições de ensino na definição da orientação concetual dos enfermeiros, pode justificar o destaque da concordância dos enfermeiros da Região Centro com as concepções de Dorothea Orem. ${ }^{17}$

Relativamente às concepções de enfermagem, saúde e ambiente da teórica, a concordância foi superior nos participantes que trabalham em serviços de medicina e especialidades médicas e cirurgia e especialidades cirúrgicas, e que exercem a sua atividade profissional como enfermeiros especialistas. Nos referidos serviços, a ênfase em uma prática centrada no autocuidado e nas capacidades da pessoa para satisfazer as necessidades e adquirir independência para o autocuidado, exige uma ajuda profissional sistematizada e intencional, 
muito centrada nesse foco de enfermagem, ${ }^{2}$ o que efetivamente tem vindo a ser concretizado de forma mais evidente pelos enfermeiros especialistas.

Embora os enfermeiros de cuidados gerais entendam a relevância de uma atuação baseada em conhecimento específico de Enfermagem, os enfermeiros especialistas, na sequência da formação avançada que efetuaram, habitualmente demonstram mais segurança na tomada de decisão, assim como maior capacidade para um olhar integral da pessoa e individualização das respetivas necessidades, aspetos centrais à promoção do autocuidado, à reconstrução da autonomia e ao bem-estar..$^{18}$

Ainda em consonância com o referencial teórico de Orem, nomeadamente com a Teoria dos Sistemas de Enfermagem, a ajuda profissional do enfermeiro pode pautar-se num sistema totalmente compensatório, sistema parcialmente compensatório ou sistema de apoioeducação. ${ }^{9,19}$ Neste contexto, em função das necessidades e das capacidades da pessoa no desempenho das atividades inerentes ao autocuidado, a intervenção do enfermeiro, implementada de forma terapêutica, tem em vista a capacitação da pessoa para a concretização do máximo de atividades que integram os diferentes domínios do autocuidado. ${ }^{20}$

A preocupação do enfermeiro especialista em progredir eficazmente para um sistema que garanta essa capacitação efetiva da pessoa na concretização do autocuidado, justifica a elevada concordância destes profissionais com as concepções de Dorothea Orem. Acresce que a intervenção educacional de suporte na capacidade de autocuidado dos clientes e a otimização do ambiente para a promoção do bem-estar, não só concorrem para a satisfação das pessoas, como garantem a qualidade da assistência de enfermagem prestada. ${ }^{9,21}$ Além disso, as Teorias de Orem orientam ações que centralizam na assistência de enfermagem a participação ativa do cuidador, vislumbrando atenção à continuidade de cuidados dos clientes no cenário hospitalar e após a alta. ${ }^{16}$

Neste seguimento, é também compreensível que a concepção de ambiente centrada nos fatores externos que afetam as capacidades de autocuidado, obtenha maior concordância nos 
Prática profissional no contexto hospitalar: visão de enfermeiros sobre contribuições... | 16

enfermeiros especialistas e nos que trabalham em serviços de medicina e especialidades médicas e cirurgia e especialidades cirúrgicas, o que se coaduna com uma prática centrada na identificação e resolução dos fatores que possam comprometer a capacidade para o autocuidado, sejam eles do domínio cognitivo, físico, emocional, psicossocial e/ou comportamental.8,20

Orem entende a pessoa e o ambiente como uma unidade única, acreditando que todos os seres humanos estão dispostos a ocupar-se de si e dos seus familiares dependentes, tendo potencial e capacidade de aprender a satisfazer as suas necessidades de autocuidado., ${ }^{8}, 10$ Contudo, quando as necessidades de autocuidado ultrapassam a capacidade de autocuidado, as pessoas vivenciam desvios de saúde, necessitando de ajuda, sendo que é neste contexto que o enfermeiro se assume como agente terapêutico. ${ }^{710}$

Para que seja possível a concretização plena do autocuidado, Orem alerta para a necessidade do envolvimento e da motivação da pessoa, aspetos fundamentais na sua responsabilização, ${ }^{8}$ o que efetivamente não é possível nos clientes internados em unidades de medicina intensiva e urgência, contextos em que a condição clínica dos clientes inviabiliza a consideração da sua intencionalidade, o seu envolvimento nos cuidados agudos e de reabilitação, bem como, globalmente, o enfoque no autocuidado. O fato de nos serviços de medicina e especialidades médicas estarem frequentemente internados clientes com elevadas exigências de autocuidado, mas simultaneamente com potencial de reconstrução de autonomia, justifica que a concordância com a concepção de pessoa de Dorothea Orem seja mais elevada nos enfermeiros destes contextos.

Em síntese, dos contributos deste estudo, fica a certeza de que vários aspetos contidos no referencial teórico de Dorothea Orem são fortemente compatíveis com os desígnios do exercício profissional dos enfermeiros, e especialmente dos enfermeiros especialistas nos contextos de medicina e especialidades médicas e cirurgia e especialidades cirúrgicas. 
A facilidade de aproximar as concepções de Orem à realidade do exercício profissional dos enfermeiros, e particularmente dos enfermeiros especialistas, de que são exemplo os enfermeiros especialistas em enfermagem de reabilitação, vem, mais uma vez, dar a oportunidade para se refletir na potencialidade das concepções desta Teórica passarem a integrar os modelos de exercício profissional de enfermagem em uso. ${ }^{12}$ Conforme defendem alguns autores, o referencial teórico de Orem deve ser usado continuamente para orientar a prática e a pesquisa de enfermagem, evidenciando ainda mais a sua aplicação e utilidade.7,14

Embora a escassa produção científica sobre as teorias de enfermagem, nomeadamente sobre as que mais contribuem para a prática dos enfermeiros, tivesse dificultado a discussão dos resultados, anseia-se que esta investigação possa desencadear a reflexão sobre esta temática e constitua mais uma oportunidade de clarificar as dúvidas quanto à relevância dos referenciais teóricos na sustentação da prática profissional dos enfermeiros. Contudo, ainda são imprescindíveis outras abordagens que permitam perceber com mais profundidade a perceção dos enfermeiros sobre as concepções de Dorothea Orem e as reais circunstâncias do seu contributo para a prática profissional, o que efetivamente constituiu uma limitação deste estudo.

\section{Conclusão}

Atendendo a que só a conexão entre teoria e prática poderão garantir a qualidade dos cuidados de enfermagem, é cada vez mais relevante o reconhecimento e a apropriação das teorias de enfermagem, bem como o potencial das mesmas subsidiarem a prática profissional. Neste contexto, é urgente que os enfermeiros sustentem o seu exercício profissional em referenciais teóricos que se adequem às necessidades dos clientes e à essência da Enfermagem.

Neste estudo, a elevada concordância com as concepções de enfermagem, pessoa, saúde e ambiente de Dorothea Orem, traduz a utilização do referencial da teorista como orientação concetual para a prática dos enfermeiros no contexto hospitalar. O fato dos enfermeiros 
Prática profissional no contexto hospitalar: visão de enfermeiros sobre contribuições... $\mid 18$

especialistas e dos que trabalham na medicina e especialidades médicas ou cirurgia e especialidades cirúrgicas, terem evidenciado uma concordância superior com as concepções de Orem, reforça a preocupação desses profissionais, e especificamente nesses contextos de cuidados, em dar enfoque à capacitação efetiva da pessoa na concretização do autocuidado.

Cabe ponderar também a influência das três teorias de Dorothea Orem, bem como o seu uso concomitante em diferentes cenários hospitalares, contudo o estudo não aprofundou o debate nessa direção, sendo o referido um possível elemento para potenciais investigações.

\section{Referências}

1. Santos BP, Sá FM, Pessan JE, Criveralo LR, Bergamo LN, Gimenez VC, et al. The training and praxis of the nurse in the light of nursing theories. Rev Bras Enferm. 2019;72(2):566-570. Doi: 10.1590/00347167-2018-0394

2. Silva NRF, Farias DC, Sousa JR, Bezerra FMC, Ferreira LS, Carvalho PMG. Nursing theories applied in the care of oncological patients: contribution to the nurse's clinical practice. Rev UNINGÁ [Internet]. 2018 [cited 2020 Jul 30];55(2):59-71. Available from: http://revista.uninga.br/index.php/uninga/article/view/1385/1685

3. Brandão MAG, Barros ALBL, Primo CC, Bispo GS, Lopes ROP. Nursing theories in the conceptual expansion of nursing practices. Rev Bras Enferm. 2019;72(2):577-81. Doi: 10.1590/0034-7167-2018-0395

4. Queirós, PJP. O conhecimento em enfermagem e a natureza dos seus saberes. Esc. Anna Nery Rev. Enferm. 2016;20(3):1-2. Doi: 10.5935/1414-8145.20160079

5. Silva ESP, Figueiredo JV, Dutra PA, Maia SRT, Prado RFS, Borrajo APC, et al. Theory of support as orem self care for nursing clinical care women mastectomized. Braz. J. of Develop. 2020;6(6):3974039750. Doi: 10.34117/bjdv6n6-496

6. Merino MFGL, Silva PLAR, Carvalho MDB, Pelloso SM, Baldissera VDA, Higarashi IH. Nursing theories in professional training and practice: perception of postgraduate nursing students. Rev Rene. 2018;19:e3363. Doi: 10.15253/2175-6783.2018193363

7. Wills EM. Grandes teorias da enfermagem baseadas nas necessidades humanas. In: McEwen M, Wills EM, organizadores. Bases Teóricas de Enfermagem. 4르 ed. Porto Alegre: Artmed; 2016. p. 133-161.

8. Orem DE. Nursing: Concepts of practice. 6를 ed. St. Louis, MO: Mosby; 2001. 
9. Hernández YC, Pacheco JAC, Larreynaga MR. La teoría The self-care deficit nursing theory: Dorothea Elizabeth Orem. Gac. méd. espirit [Internet]. 2017 [cited 2020 Jul 29];19(3):1-11. Available from: https://www.medigraphic.com/pdfs/espirituana/gme-2017/gme173i.pdf

10. Santos B, Ramos A, Fonseca C. Training to practice: Importance of Self-Care Theory in Nursing Process for improving care. J Aging Innovation [Internet]. 2017 [cited 2020 Aug 09];6(1):51-54. Available from: http://journalofagingandinnovation.org/wp-content/uploads/6-Autocuidadoforma $\%$ C $3 \%$ A $7 \%$ C $3 \%$ A3o.pdf

11. Garcia TR. Professional language and nursing domain. Texto \& contexto enferm. 2019;28:e20190102. Doi: 10.1590/1980-265x-tce-2019-0001-0002

12. Ribeiro OMPL, Martins MMFPS, Tronchin DMR, Forte ECN. The perspective of portuguese nurses on nursing metaparadigmatic concepts. Texto \& contexto enferm. 2018;27(2):e3970016. Doi: 10.1590/0104-070720180003970016.

13. Ribeiro OMPL. Contextos da prática hospitalar e conceções de enfermagem: Olhares sobre o real da qualidade e o ideal da excelência no exercício profissional dos enfermeiros [tese]. Porto: Instituto de Ciências Biomédicas Abel Salazar, Universidade do Porto; 2018.

14. Younas A. A Foundational Analysis of Dorothea Orem's Self-Care Theory and Evaluation of Its Significance for Nursing Practice and Research. Creat Nurs. 2017;23(1):13-23. Doi: 10.1891/10784535.23.1.13

15. Hagran A, Fakharany A. Critique of Orem's theory. J. Middle East North Afr. sci [Internet]. 2015 [cited 2020 Aug 15];1(5):12-17. Available from: https://pdfs.semanticscholar.org/5761/17009ae992dc2e2ee652cb66c03020d633f2.pdf

16. Demoro CCS, Fontes CMB, Trettene AS, Cianciarullo TI, Lazarini IM. Applicability of Orem: training of caregiver of infant with Robin Sequence Rev Bras Enferm. 2018;71(Suppl 3):1469-1473. Doi: 10.1590/0034-7167-2016-0562

17. Ribeiro OMPL, Martins MMFPS, Tronchin DMR, Silva JMAV. Professional nursing practice grounded in the theoretical framework of the discipline: reality or utopia. Rev Enf Ref. 2018;IV(19):3948. Doi: 10.12707/RIV18040

18. Silva RMO, Luz MDA, Fernandes JD, Silva LS, Cordeiro ALAO, Mota LSR. Becoming a specialist: portuguese nurses`expectations after completing the specialization program. Rev Enf Ref. 2018;IV(16):147-154. Doi: 10.12707/RIV17076

19. Pires AF, Santos BN, Santos PN, Brasil VR, Luna AA. A importância da teoria do autocuidado de Dorothea E. Orem no cuidado de enfermagem. Rev Rede cuid saúde [Internet]. 2015 [acesso 2020 Ago 21];9(2):1-4. Disponível em: http://publicacoes.unigranrio.edu.br/index.php/rcs/article/view/2533/1292 
Prática profissional no contexto hospitalar: visão de enfermeiros sobre contribuições... $\mid 20$

20. Petronilho F, Machado M. Teorias de Enfermagem e Autocuidado: Contributos para a Construção do Cuidado de Reabilitação. In: Marques-Vieira C, Sousa L, organizadores. Cuidados de Enfermagem de Reabilitação à Pessoa ao Longo da Vida. Loures: Lusodidacta; 2016. p. 3-12.

21. Mohammadpour A, Sharghi NR, Khosravan S, Alami A, Akhond M. The effect of a supportive educational intervention developed based on the Orem's self-care theory on the self-care ability of patients with myocardial infarction: A randomized controlled trial. J Clin Nurs. 2015; 24(11-12):168692. Doi: $10.1111 /$ jocn. 12775

\section{Editora Científica: Tânia Solange Bosi de Souza Magnago \\ Editora Associada: Rafaela Andolhe}

\section{Autor correspondente}

Olga Maria Pimenta Lopes Ribeiro

E-mail: olgaribeiro@esenf.pt

Endereço: Rua Dr. António Bernardino de Almeida

CEP: 4200-072 Porto, Portugal

\section{Contribuições de Autoria}

\section{1 - Olga Maria Pimenta Lopes Ribeiro}

Concepção ou desenho do estudo/pesquisa, análise e/ou interpretação dos dados, revisão final com participação crítica e intelectual no manuscrito.

\section{2 - Letícia de Lima Trindade}

Análise e/ou interpretação dos dados, revisão final com participação crítica e intelectual no manuscrito.

\section{3 - João Miguel Almeida Ventura da Silva}

Análise e/ou interpretação dos dados, revisão final com participação crítica e intelectual no manuscrito.

\section{4 - Ana da Conceição Alves Faria}

Análise e/ou interpretação dos dados, revisão final com participação crítica e intelectual no manuscrito.

\section{Como citar este artigo}

Ribeiro OMPL, Trindade LL, Silva JMAV, Faria ACA. Prática profissional no contexto hospitalar: visão de enfermeiros sobre contribuições das concepções de Dorothea Orem. Rev. Enferm. UFSM. 2021 [Acesso em: Anos Mês Dia]; vol.11 e28: 1-20. DOI: https: //doi.org/10.5902/2179769254723 